\title{
Design of Smart Light Source based on Bi-color LED with Single Duty Cycle for Correlated Color Temperature Adjustment
}

\section{Lingyuan Qiao}

Dalian Polytechnic university

Huanyue Zhang

Dalian University of Technology

Jingjie Yu

Dalian Polytechnic University

Fan Cao

Dalian Polytechnic University

Yingming Gao ( $\nabla$ gyming@dlpu.edu.cn )

Dalian Polytechnic University

\section{Research Article}

Keywords: LED, correlated color temperature, pulse width modulation, duty cycle

Posted Date: July 8th, 2021

DOI: https://doi.org/10.21203/rs.3.rs-597067/v1

License: (c) (1) This work is licensed under a Creative Commons Attribution 4.0 International License. Read Full License 


\section{Declarations}

Competing Interests: All authors certify that they have no affiliations with or involvement in any organization or entity with any financial interest or non-financial interest in the subject matter or materials discussed in this manuscript.

Funding Information: This work was supported by Scientific research project of education department of Liaoning province(J2020019).

Author contributions: All authors contributed to the study conception and design. Material preparation, data collection and analysis were performed by Yingming Gao and Lingyuan Qiao. The first draft of the manuscript was written by Lingyuan Qiao and all authors commented on the manuscript.

Consent to Participate: All the authors participated in the study and approved the final manuscript. Consent to Publish: Our work is original and has not been published elsewhere in any form. All authors have agreed to publish in Optical and Quantum Electronics. 


\title{
Design of smart light source based on bi-color LED with single duty cycle for correlated color temperature adjustment
}

\author{
Lingyuan Qiao a , Huanyue Zhang ${ }^{b}$, Jingjie Yu ${ }^{\text {a }}$, Fan Cao ${ }^{a}$, ${ }^{*}$ ingming Gao ${ }^{a, b}$ \\ ${ }^{a}$ Dalian Polytechnic University, Department of Information Science and Engineering, 1 Chigong Yuan, Ganjingzi \\ District, Dalian City 116034, China \\ ${ }^{b}$ Dalian University of Technology, Instrumental Analysis and Research Center, 2 Linggong Road, Ganjingzi \\ District, Dalian City 116024, China
}

\begin{abstract}
Color-adjustable light sources facilitate both mood lighting and daylight harvesting. A single duty cycle can be used by a bi-color LED to adjust the correlated color temperature by associating it with the duty cycle of the pulse width modulation dimming signal of the cold and warm light sources. The one-to-one mapping relationship between the single duty cycle and the correlated color temperature is based on the color mixing theory of bi-color LEDs. A method to correlate the dimming signals for cold and warm LEDs is presented. The influence of the time characteristics of the two basic signals on dimming and color temperature adjustment is analyzed. The dimming system of bi-color LEDs is designed, and the method used to adjust the correlated color temperature with a single duty cycle is verified. The experiment showed that the correlated color temperature can be accurately adjusted by the proposed method.
\end{abstract}

Keywords: LED, correlated color temperature, pulse width modulation, duty cycle.

*Yingming Gao, E-mail: gyming@ dlpu.edu.cn

\section{Introduction}

Previous studies have shown that lighting color temperature and illumination can change people's visual perception and emotion; they also play an important role in the control of human endocrine system activity, physiological rhythm, and psychological cognition [1-4]. Existing literature [5] has identified that the light irradiation of highly correlated color temperature light-emitting diodes (LEDs) has an impact on sleep, mood, and physical strength. Moreover, people exposed to cold light experience better sleep quality and quantity than those exposed to warm light. A previous study [6] indicated that the interaction between illumination and color temperature affects cognition and self-control. In addition to mood lighting, which affects the emotional feeling of humans, color-tunable light sources may be used for daylight harvesting. The correlated color temperature of daylight can vary from $2000 \mathrm{~K}$ at sunrise to $5000 \mathrm{~K}$ for direct daylight at noon, even exceeding $10000 \mathrm{~K}$ under overcast conditions. Along with the correlated color temperature, 
the illuminance of daylight also varies dynamically. Therefore, to create a near-exact visual sensation and energy-efficient lighting, an artificial light source (visually matched LED lamp) with independently tunable correlated color temperature and illuminance should preferably be introduced in daylight harvesting schemes [7,8].

As is widely known, white light with different correlated color temperatures can be produced by a blue LED or near-ultraviolet LED stimulating proper yellow phosphors. For RGB three-color [9-12] or RGBA four-color LEDs [13], the Grassmann color law is applied to adjust the ratio of the luminosity of different color LEDs to produce white light. These types of LED systems require three or more pulse-width-modulated (PWM) output channels, which make them difficult to practically realize. Among the many correlated color temperature regulation methods, the double white LED method is more convenient and practical than others. The PWM dimming method is used to adjust the luminous flux of high- and low-correlated color temperature LEDs and then mix them together to adjust the correlated color temperature [14-17]. We use the bi-color LED color matching method to simplify the implementation of correlated color temperature regulation. Through association of the dimming duty cycle of cold and warm LEDs, we transform the mapping relationship between the duty cycle and the correlated color temperature from two duty cycles to a single duty cycle.

This paper is organized as follows. Section 2 describes the principle of correlating the duty cycle of warm and cold LED dimming signals. Section 3 defines dimming signals for warm and cold LEDs, analyzes the time relevant character of dimming signals for precision of color adjustment, and designs a control system for bi-color LED. Section 4 verifies the method through a series of experiment. Conclusions and future research are presented in Sec. 5. 


\section{Correlated Color Temperature Calculation Model of Bi-color LED}

The correlated color temperature represents the color attribute of light that has the temperature of blackbody radiation. According to the principle of color matching, the essence of color matching with bi-color LEDs is to change the ratio of cold light luminosity to warm light luminosity using dimming technology to adjust the correlated color temperature [18]. We used the correlated color temperature calculation model proposed by Ref. [19], which is shown in Eq. (1):

$$
\left\{\begin{array}{c}
C C T=-449 n^{3}+3525 n^{2}-6823.3 n+5520.33 \\
n=\frac{x_{m}-0.3320}{y_{m}-0.1858}
\end{array}\right.
$$

where $n$ is the inverse slope, and $x_{m}$ and $y_{m}$ are the color coordinates of the mixed light, which can be obtained by

$$
\left\{\begin{array}{l}
x_{m}=\frac{R_{c} D_{c} x_{c}+R_{w} D_{w} x_{w}}{R_{c} D_{c}+R_{w} D_{w}} \\
y_{m}=\frac{R_{c} D_{c} y_{c}+R_{w} D_{w} y_{w}}{R_{c} D_{c}-R_{w} D_{w}}
\end{array}\right.
$$

where $R_{c}=\frac{\Phi_{c}}{y_{c}}$, and $R_{w}=\frac{\Phi_{w}}{y_{w}}$.

According to Eqs. (1) and (2), two duty cycles are required to obtain the correlated color temperature: the dimming duty cycles $D_{c}$ and $D_{w}$ of the cold and warm light sources, respectively. They are independent and have no relation to each other, which makes it more difficult to determine the correlated color temperature [20]. In a previous study [21], two complementary PWM signals with equal periods were used to adjust the cold and warm light source, respectively, and the duty cycle of the two PWM signals met the condition $D_{c}+D_{w}=1$. However, the study did not conduct a theoretical analysis and did not establish the mapping relationship between the duty cycle and values of the photometry; thus, the correlated color temperature could not be accurately controlled. Substituting $1-D_{w}$ for $D_{c}$ into Eq. (2), we obtain the mapping relationship between the correlated color temperature and warm light dimming 
duty cycle $D_{w}$, as shown in Eq. (3). Clearly, it is more convenient to confirm the correlated color temperature using a single duty cycle compared with two independent dimming duty cycles.

$$
\left\{\begin{aligned}
C C T= & -449 n^{3}+3525 n^{2}-6823.3 n+5520.33 \\
n= & \frac{\frac{R_{C}\left(1-D_{w}\right) x_{c}+R_{w} D_{w} x_{w}}{R_{C}\left(1-D_{w}+R_{w} D_{w}\right.}-0.3320}{\frac{R_{c}\left(1-D_{w}\right) y_{c}+R_{w} D_{w} y_{w}}{R_{c}\left(1-D_{w}\right)-R_{w} D_{w}}-0.1858} .
\end{aligned}\right.
$$

Using Eq. (3), we present the curve between the correlated color temperature and the dimming duty cycle $D_{w}$ of a warm white LED in Fig. 1. The relationship between the correlated color temperature and $D_{w}$ is nonlinear. The correlated color temperature decreases with an increase in the warm duty cycle $D_{w}$; it is lower than that of a cold LED but higher than that of a warm LED. The parameters of the cold and warm LEDs used to obtain Fig.1 are listed in Table 1.

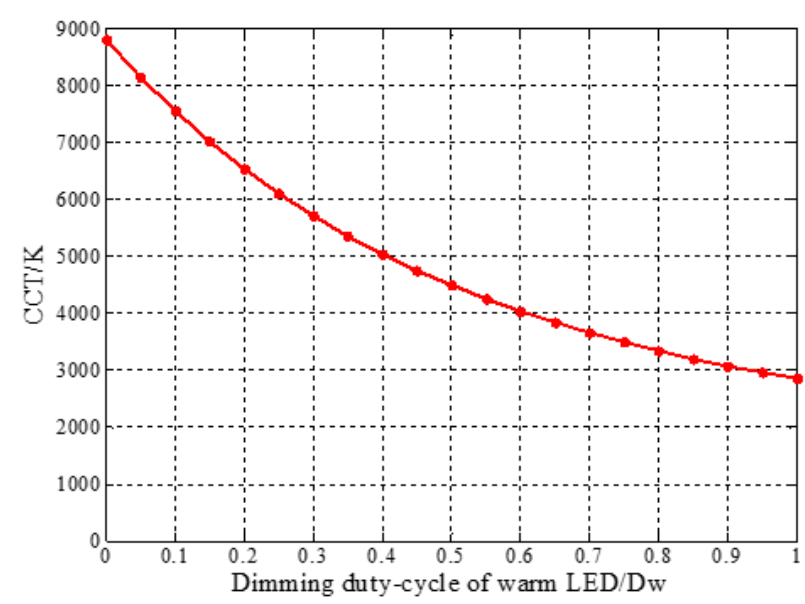

Fig. 1 Relationship between duty cycle $D_{w}$ and the correlated color temperature (CCT)

\section{Dimming Signals}

\subsection{Definition of Dimming Signals}

According to the Kruithof curve, a comfortable lighting environment can be obtained only through the reasonable combination of the correlated color temperature and illumination. A smart light source is characterized by its ability to adjust both the correlated color temperature and illumination, which is convenient for application in different environments, and achieves the goal 
of implementing one multi-use lamp. To simultaneously adjust the luminous flux and correlated color temperature of the light source, two basic PWM signals, $\mathrm{PWM}_{1}$ and $\mathrm{PWM}_{2}$, are defined; their periods are $T_{1}$ and $T_{2}$, respectively. The duty cycles $\mathrm{D}$ and $\mathrm{d}$, corresponding to $\mathrm{PWM}_{1}$ and $\mathrm{PWM}_{2}$, respectively, are shown in Fig. 2.

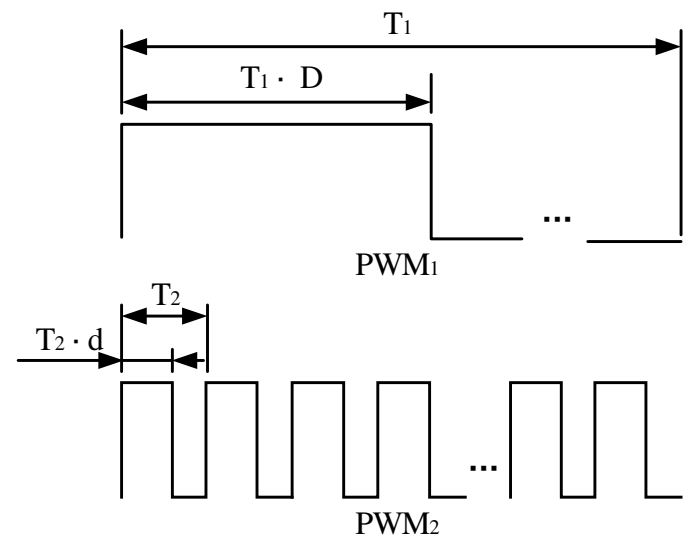

Fig. 2 Definition of the two basic PWM signals

Using the two basic PWM signals, the generation of the dimming signals introduced in this study is shown in Fig. 3. The dimming signal of the warm LED is $\mathrm{PWM}_{w}=\mathrm{PWM}_{1} \cap \mathrm{PWM}_{2}$, and that of the cold LED is $\mathrm{PWM}_{c}=\mathrm{PWM}_{1} \cap \overline{\mathrm{PWM}_{2}}$. When $\mathrm{PWM}_{1}=1, \mathrm{PWM}_{w}=\mathrm{PWM}_{2}$ and $\mathrm{PWM}_{c}=\overline{\mathrm{PWM}_{2}}$. Clearly, if the duty cycle $\mathrm{D}_{w}$ of $\mathrm{PWM}_{w}$ is $d$, then the duty cycle $\mathrm{D}_{c}$ of $\mathrm{PWM}_{c}$ is $1-d$, and the two duty cycles are associated. In particular, when $\mathrm{PWM}_{1}=0$, then $\mathrm{PWM}_{w}=\mathrm{PWM}_{c}=0$.

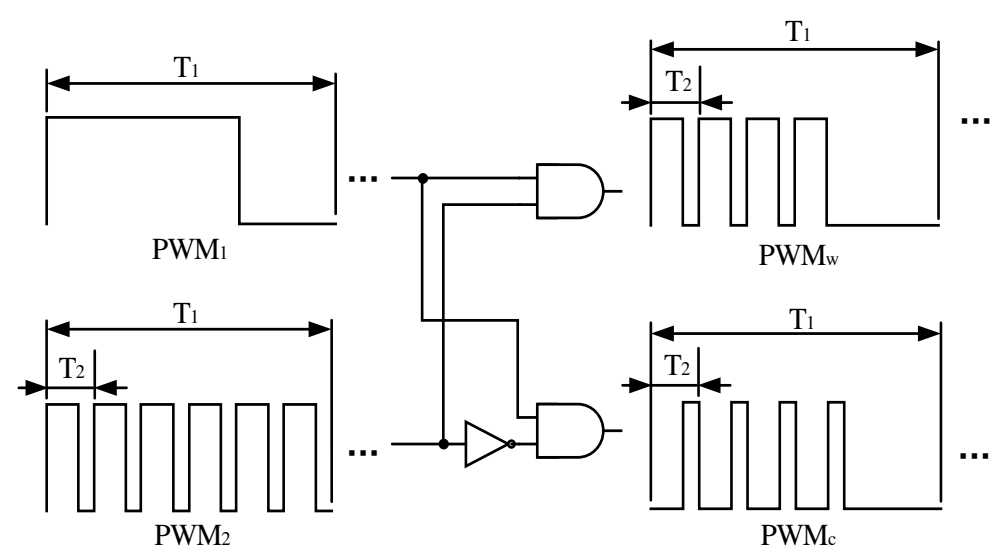

Fig. 3 Generation of dimming signals for cold and warm LEDs 


\subsection{Analysis of Dimming Signals}

The two basic signals $\mathrm{PWM}_{1}$ and $\mathrm{PWM}_{2}$ should satisfy the time-related constraint, or the precision of the color adjustment may deteriorate. According to the definition presented in Sec. 3.1, when $\mathrm{PWM}_{1}$ is high voltage, the signals $\mathrm{PWM}_{w}$ and $\mathrm{PWM}_{c}$, which are used to dim the warm and cold light, respectively, are output. The relationship between the high voltage duration of signal $\mathrm{PWM}_{1}$ and cycle $\mathrm{T}_{2}$ of $\mathrm{PWM}_{2}$ can be expressed by Eq. (4):

$$
\mathrm{T}_{1} \cdot \mathrm{D}=\mathrm{k} \cdot \mathrm{T}_{2} \pm \mathrm{t}\left(\mathrm{k}=1,2, \cdots ; 0 \leq \mathrm{t}<\mathrm{T}_{2}\right),
$$

where $t$ represents a time period less than $\mathrm{T}_{2}$ and $\mathrm{k}$ is the number of cycles of $\mathrm{T}_{2}$ that are composed of the high voltage period of the $\mathrm{PWM}_{1}$ signal. According to Eq. (4), if $t=0, \mathrm{~T}_{1} \cdot \mathrm{D}$ is equal to $\mathrm{T}_{2}$ multiplied by integer $k$. Then, the duration of the warm light emission $t_{w}$ is $\mathrm{k} \cdot \mathrm{T}_{2} \cdot$ $\mathrm{d}$, and that of the cold light emission $\mathrm{t}_{\mathrm{c}}$ is $\mathrm{k} \cdot \mathrm{T}_{2} \cdot(1-\mathrm{d})$ in one cycle of $\mathrm{PWM}_{1}$. We set $\Phi_{c}$ and $\Phi_{w}$, which are the luminous flux outputs corresponding to the constant working current of the cold and warm LEDs, respectively.

$\Phi_{w_{-} t}$ is the dimming luminous flux of the warm light:

$$
\Phi_{w_{-} t}=\frac{\Phi_{w} \cdot t_{w}}{\mathrm{~T}_{1}}=\frac{\Phi_{w} \cdot \mathrm{k} \cdot \mathrm{T}_{2} \cdot \mathrm{d}}{\mathrm{T}_{1}} .
$$

$\Phi_{c_{-} t}$ is the dimming luminous flux of the cold light:

$$
\Phi_{c_{-} t}=\frac{\Phi_{c} \cdot t_{c}}{\mathrm{~T}_{1}}=\frac{\Phi_{c} \cdot \mathrm{k} \cdot \mathrm{T}_{2} \cdot(1-\mathrm{d})}{\mathrm{T}_{1}} .
$$

The ratio of $\Phi_{w_{-} t}$ to $\Phi_{c_{-} t}$ determines the mixed color:

$$
\frac{\Phi_{w_{-} t}}{\Phi_{c_{-} t}}=\frac{\Phi_{w} \cdot \mathrm{d}}{\Phi_{C}(1-\mathrm{d})} .
$$

The mixed luminous flux is

$$
\Phi_{m}=\Phi_{w} \cdot \mathrm{D} \cdot \mathrm{d}+\Phi_{c} \cdot \mathrm{D} \cdot(1-\mathrm{d}) .
$$

It can be concluded from Eqs. (7) and (8) that the ratio of the luminous flux of cold light to that of warm light, i.e., the total luminous flux $\Phi_{m}$, is independent of the periods $T_{1}$ and $T_{2}$. 
However, it is affected by the luminous properties of the light source and the duty cycle of the dimming signal when the high voltage duration of $\mathrm{PWM}_{1}$ is equal to an integer multiple of $\mathrm{T}_{2}$, which is the period of signal $\mathrm{PWM}_{2}$. In particular, when the luminous flux values $\Phi_{C}$ and $\Phi_{W}$, which correspond to the cold and warm light sources, respectively, are the same, the mixed luminous flux is independent of the duty cycle of the $\mathrm{PWM}_{2}$ signal and is equal to $\Phi_{W} \cdot \mathrm{D}$ or $\Phi_{C} \cdot \mathrm{D}$, which is affected only by the duty cycle of $\mathrm{PWM}_{1}$. This makes it possible to adjust the correlated color temperature while maintaining a constant illumination, which may be convenient for daylight-responsive lighting.

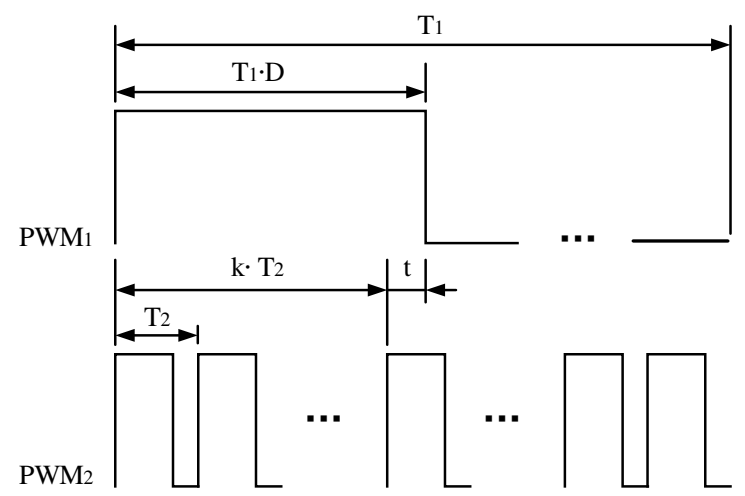

Fig. 4 Illustration of one state of Eq. (4)

If $t$ is nonzero, a possible state described by Eq. (4) is illustrated in Fig. 4. Considering $\mathrm{PWM}_{w}=\mathrm{PWM}_{2}$ and $\mathrm{PWM}_{c}=\overline{\mathrm{PWM}_{2}}$, when $\mathrm{PWM}_{1}=1$, the duration $t_{w}$ of the warm light emission is $\mathrm{k} \cdot \mathrm{T}_{2} \cdot \mathrm{d}+\mathrm{t}$, and the duration $t_{c}$ of the cold light emission is $\mathrm{k} \cdot \mathrm{T}_{2} \cdot(1-\mathrm{d})$.

$\Phi_{w_{-} t}$ is the dimming luminous flux of the warm light:

$$
\Phi_{w_{-} T}=\frac{\Phi_{w} \cdot t_{w}}{T_{1}}=\frac{\Phi_{w} \cdot\left(\mathrm{k} \cdot \mathrm{T}_{2} \cdot \mathrm{d}+\mathrm{t}\right)}{\mathrm{T}_{1}} .
$$

$\Phi_{c_{-} t}$ is the dimming luminous flux of the cold light:

$$
\Phi_{C_{-} T}=\frac{\Phi_{C} \cdot t_{C}}{\mathrm{~T}_{1}}=\frac{\Phi_{C} \cdot \mathrm{k} \cdot \mathrm{T}_{2} \cdot(1-\mathrm{d})}{\mathrm{T}_{1}} .
$$

The ratio of $\Phi_{w_{-} T}$ to $\Phi_{c_{-} T}$, which determines the precision of the mixed color, is 


$$
\frac{\Phi_{w_{-} t}}{\Phi_{c_{-} t}}=\frac{\Phi_{w} \cdot\left(\mathrm{k} \cdot \mathrm{T}_{2} \cdot \mathrm{d}+\mathrm{t}\right)}{\Phi_{c} \cdot \mathrm{k} \cdot \mathrm{T}_{2} \cdot(1-\mathrm{d})}=\frac{\Phi_{w} \cdot\left(\mathrm{d}+\frac{\mathrm{t}}{\mathrm{k} \cdot \mathrm{T}_{2}}\right)}{\Phi_{c} \cdot(1-\mathrm{d})} .
$$

It is clear that Eq. (11) is influenced by $k$ and $t$. Considering $\frac{t}{T_{2}}<1, \frac{\mathrm{t}}{\mathrm{k} \cdot \mathrm{T}_{2}}$ would be close to zero with an increase in $\mathrm{k}$. Given $\mathrm{T}_{1}$ and Eq. (4) and considering that $k$ is the number of cycles of $\mathrm{T}_{2}$ that are composed of the high voltage duration of $\mathrm{PWM}_{1}$, the frequency of $\mathrm{PWM}_{2}$ should increase with an increase in $k$. It can be concluded that Eq. (11) is independent of $t$, and it converges to $\frac{\Phi_{w} \cdot \mathrm{d}}{\Phi_{c}(1-\mathrm{d})}$ as the frequency of $\mathrm{PWM}_{2}$ increases.

The mixed luminous flux $\Phi_{m}=\Phi_{w_{-} t}+\Phi_{c_{-} t}$ can be expressed as

$$
\Phi_{m}=\frac{\Phi_{w} \cdot\left(\mathrm{k} \cdot \mathrm{T}_{2} \cdot \mathrm{d}+\mathrm{t}\right)}{\mathrm{T}_{1}}+\frac{\Phi_{c} \cdot \mathrm{k} \cdot \mathrm{T}_{2} \cdot(1-\mathrm{d})}{\mathrm{T}_{1}}
$$

If the luminous flux $\Phi_{c}$ is the same as $\Phi_{w}, \Phi_{m}$ can be expressed as $\Phi_{w} \cdot \frac{\left(k T_{2}+t\right)}{T_{1}}$ or $\Phi_{c}$. $\frac{\left(k T_{2}+t\right)}{T_{1}}$. According to Eq. (4), $k T_{2}+t$ is equal to $\mathrm{T}_{1} \cdot \mathrm{D}$, and the mixed luminous flux $\Phi_{m}$ is $\Phi_{w} \cdot D$ or $\Phi_{c} \cdot D$, which is independent of both $t$ and $d$.

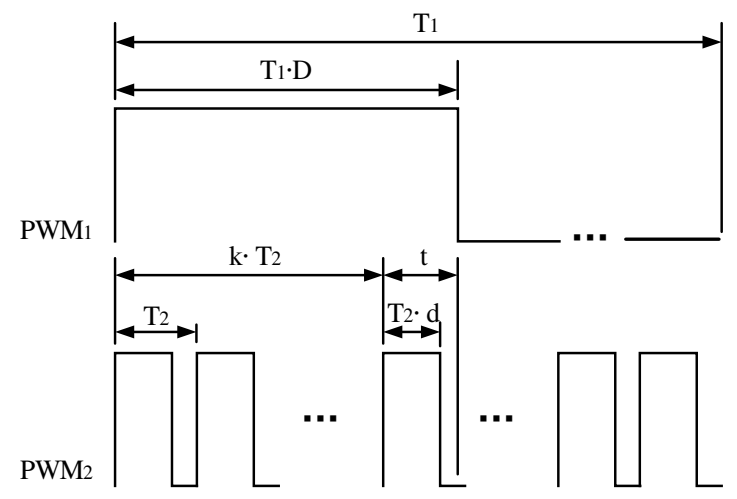

Fig. 5 Alternative illustration of Eq. (4)

Another state described by Eq. (4) is presented in Fig. 5. During the high voltage period of $\mathrm{PWM}_{1}$, the duration of the warm light emission $t_{w}$ is $\mathrm{k} \cdot \mathrm{T}_{2} \cdot \mathrm{d}+\mathrm{T}_{2} \cdot \mathrm{d}$, and the duration of the cold light emission $t_{c}$ is $\mathrm{k} \cdot \mathrm{T}_{2} \cdot(1-\mathrm{d})+\mathrm{t}-\mathrm{T}_{2} \cdot \mathrm{d}$. 
$\Phi_{w_{-} t}$ is the dimming luminous flux of the warm light:

$$
\Phi_{w_{-} t}=\frac{\Phi_{W} \cdot t_{w}}{\mathrm{~T}_{1}}=\frac{\Phi_{W} \cdot(\mathrm{k}+1) \cdot \mathrm{T}_{2} \cdot \mathrm{d}}{\mathrm{T}_{1}} .
$$

$\Phi_{c_{-} t}$ is the dimming luminous flux of the cold light:

$$
\Phi_{c_{-} t}=\frac{\Phi_{C} \cdot t_{C}}{\mathrm{~T}_{1}}=\frac{\Phi_{C} \cdot\left((\mathrm{k}-(\mathrm{k}+1) \cdot \mathrm{d}) \cdot \mathrm{T}_{2}+\mathrm{t}\right)}{\mathrm{T}_{1}} .
$$

The ratio of $\Phi_{w_{-} T}$ to $\Phi_{c_{-} T}$ is

$$
\frac{\Phi_{w_{-} t}}{\Phi_{c_{-} t}}=\frac{\Phi_{w} \cdot(\mathrm{k}+1) \cdot \mathrm{T}_{2} \cdot \mathrm{d}}{\Phi_{c} \cdot\left((\mathrm{k}-(\mathrm{k}+1) \cdot \mathrm{d}) \cdot \mathrm{T}_{2}+\mathrm{t}\right)}=\frac{\Phi_{w} \cdot \mathrm{d}}{\Phi_{c} \cdot\left(\left(\frac{\mathrm{k}}{\mathrm{k}+1}-\mathrm{d}\right)+\frac{\mathrm{t}}{(\mathrm{k}+1) \mathrm{T}_{2}}\right)} .
$$

The analysis of Eq. (15) is similar to that of Eq. (11). With an increase in the frequency of

$\mathrm{PWM}_{2}$, Eq. (15) converges to $\frac{\Phi_{w} \cdot \mathrm{d}}{\Phi_{c}(1-\mathrm{d})}$, owing to the convergence of the denominator to $\Phi_{c} \cdot(1-$ d).

The mixed luminous flux $\Phi_{m}=\Phi_{w_{-} t}+\Phi_{c_{-} t}$ can be expressed as

$$
\Phi_{m}=\frac{\Phi_{w} \cdot(\mathrm{k}+1) \cdot \mathrm{T}_{2} \cdot \mathrm{d}}{\mathrm{T}_{1}}+\frac{\Phi_{c} \cdot\left((\mathrm{k}-(\mathrm{k}+1) \cdot \mathrm{d}) \cdot \mathrm{T}_{2}+\mathrm{t}\right)}{\mathrm{T}_{1}} .
$$

Similar to the analysis of Eq. (12), it can be concluded that $\Phi_{m}$ is $\Phi_{c} \cdot D$ or $\Phi_{w} \cdot D$ when $\Phi_{c}=\Phi_{w}$. That is, the mixed luminous flux $\Phi_{m}$ is independent of both $t$ and $d$, and it is only affected by $\mathrm{D}$, which is the duty cycle of $\mathrm{PWM}_{1}$, given the same $\Phi_{c}$ and $\Phi_{w}$.

\subsection{Generation of Dimming Signals}

The dimming system of a bi-color LED smart light source consists of a power supply unit, control unit, and drive unit, as shown as in Fig. 6. The input voltage of the system is $12 \mathrm{~V}$, which was also implemented as the input voltage of the driving unit. The $12 \mathrm{~V}$ voltage is converted to $5 \mathrm{~V}$ by a power supply unit to power the control unit. The control unit is composed of a microcontroller (STC15F2K60S2, STCmicro Technology Ltd., Shenzhen, China) and gate circuit. It receives control instructions and generates dimming signals for the cold and warm lights. The drive unit 
adopts two constant current buck drivers (PT4115, H\&M Semiconductor Ltd., Shenzhen, China) and is used to drive the cold and warm LEDs. The cold and warm dimming signals generated from the control unit are respectively sent to the PWM dimming input terminals of the two buck drivers. The experimental dimming system of a bi-color LED is presented in Fig. 7.

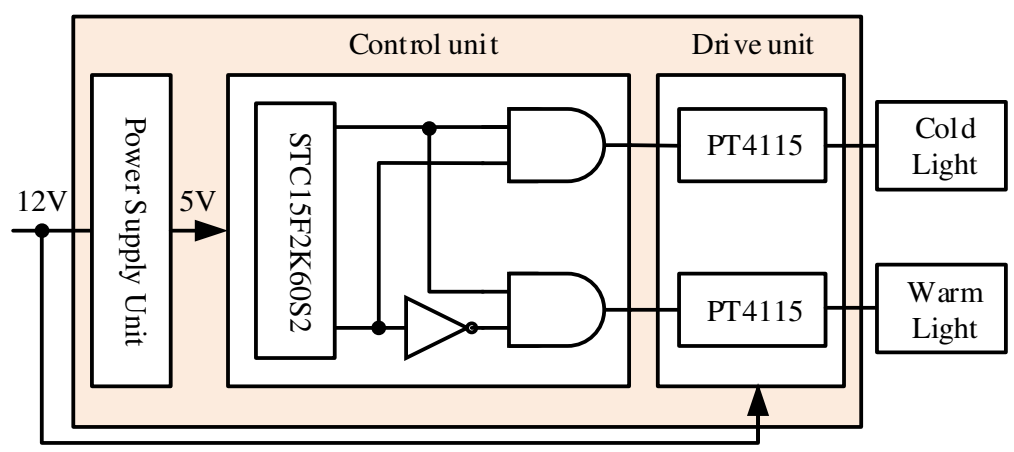

Fig. 6 Basic block diagram of bi-color LED system

We set the period of the basic dimming signal $\mathrm{PWM}_{1}$ to $1 \mathrm{~ms}$ at 10 levels; thus, the minimal duration of high voltage is $100 \mu$ s. The $\mathrm{PWM}_{2}$ signal is also at 10 levels; its period is set to 100 $\mu$ s to satisfy the requirement that the high voltage duration of $\mathrm{PWM}_{1}$ is an integer multiple of the period of $\mathrm{PWM}_{2}$, according to the discussion in Sec. 3.2. Using the timer of the microcontroller, the basic dimming signals $\mathrm{PWM}_{1}$ and $\mathrm{PWM}_{2}$ are output from $\mathrm{P} 1.0$ and P1.1, respectively, with the initial state of the high voltage.

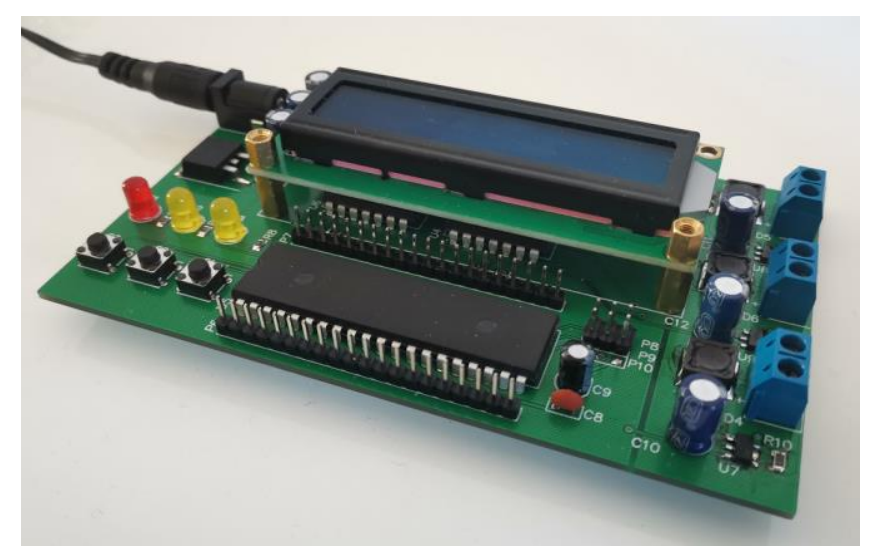

Fig. 7 Bi-color LED dimming experiment system 
Figure 8 presents a flow chart that describes how the output signals $\mathrm{PWM}_{1}$ and $\mathrm{PWM}_{2}$ are obtained through P1.0 and P1.1, respectively, by using a timer interrupt function. Two integer variables, counter 1 and counter2, are used to determine the period and duty cycle of the signals corresponding to $\mathrm{PWM}_{2}$ and $\mathrm{PWM}_{1}$, respectively. The initial and final values of the two variables are set to zero and ten, respectively. Counter1 is incremented by one every time the timer interrupt function is executed, and counter 2 is incremented by one when counter1 accumulates from zero to ten.

Given a timing interval of $10 \mu \mathrm{s}$, two PWM signals can be obtained with the same duty cycle of $50 \%$ but with different periods (200 $\mu \mathrm{s}$ and $2 \mathrm{~ms})$. When the two variables increase from zero to ten, they should be reset, and the output voltages of P1.0 and P1.1 should be reversed. If the output voltage of the pins is reversed when counter 1 or counter2 is less than ten, two PWM signals may be output with an adjustable duty cycle and periods of $100 \mu \mathrm{s}$ and $1 \mathrm{~ms}$. For example, the output voltage of the pins was reversed when counter $1=3$ and counter2=6, two PWM signals with duty cycles of $30 \%$ and $60 \%$ could be obtained. 


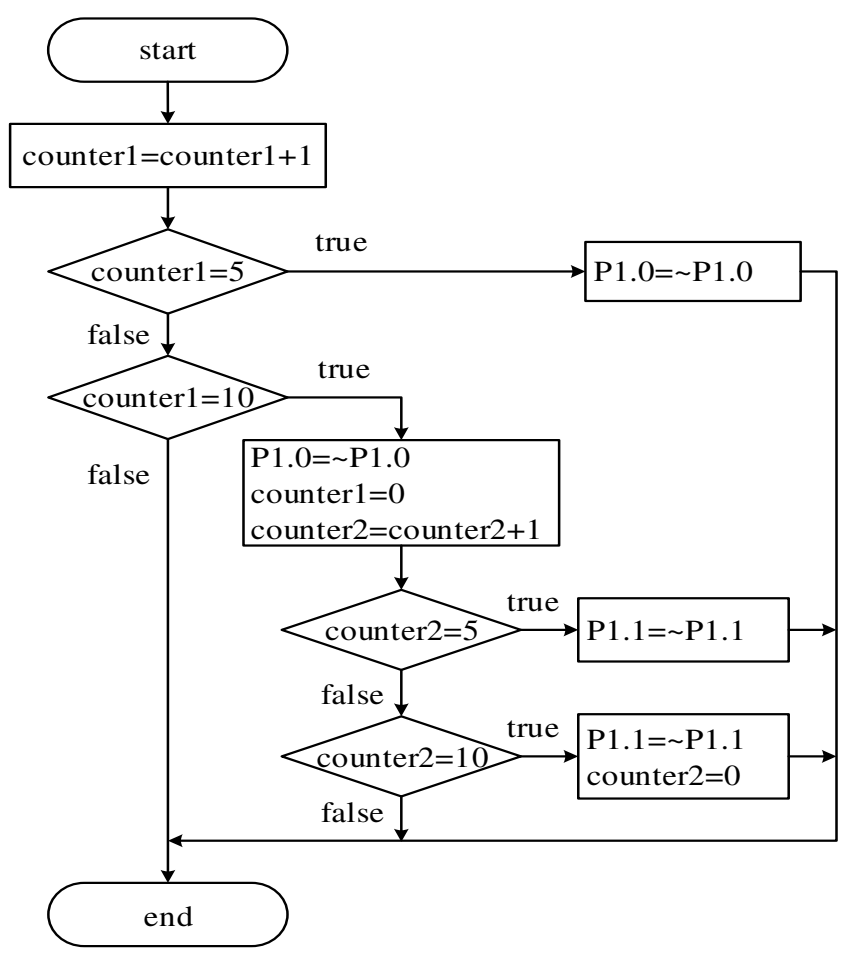

Fig. 8 Flow chart of the production of a basic dimming signal

Given a timing interval of $10 \mu \mathrm{s}$, two basic signals $\mathrm{PWM}_{1}$ and $\mathrm{PWM}_{2}$ with the same duty cycle of $50 \%$ but different periods ( $1 \mathrm{~ms}$ and $100 \mu \mathrm{s})$ are presented in Fig. 9. The signals are used to dim the cold and warm LEDs, respectively, and are obtained by the gate-circuit transformation of basic signals $\mathrm{PWM}_{1}$ and $\mathrm{PWM}_{2}$, as shown in Fig. 10.

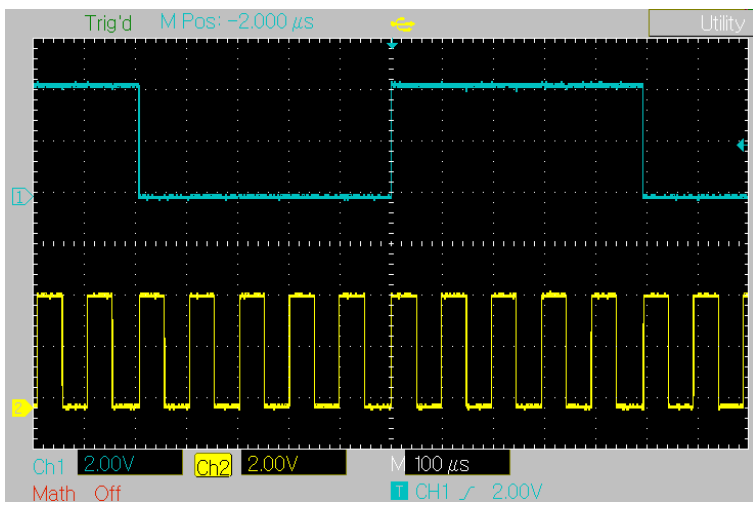

Fig. 9 Basic signals $\mathrm{PWM}_{1}$ and $\mathrm{PWM}_{2}$

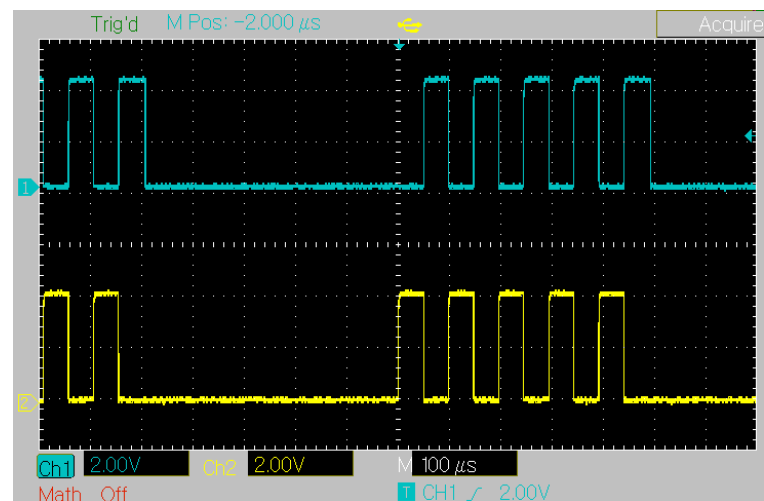

Fig. 10 Cold LED and warm LED dimming signals

\section{Experiment and Analysis}




\subsection{Test of Linearity of PWM Dimming}

The test system includes a spectral radiometer (HAAS-2000), integrating sphere, computer, and current source. Two $3 \mathrm{~W}$ LEDs, cold and warm, were placed in the integrating sphere, and their chromaticity parameters were measured with a constant current $750 \mathrm{~mA}$, as shown in Table 1 . Considering that a high PN junction temperature would cause a color drift or light decay [22,23], we welded the two LEDs on the aluminum substrate to dissipate heat to reduce the influence of temperature on the light source.

Table 1 Parameters of cold and warm LEDs

\begin{tabular}{cccc}
\hline LED & $(\mathrm{x}, \mathrm{y})$ & $\mathrm{CCT} / \mathrm{K}$ & Luminous flux/lm \\
\hline Cold white light & $(0.2901,0.2917)$ & 8800 & 179.16 \\
Warm white light & $(0.4583,0.4257)$ & 2840 & 179.69 \\
\hline
\end{tabular}

Using the system shown in Fig. 7, we obtained the corresponding relationship between the luminous flux of the LED and the dimming duty cycle, as shown in Fig. 11. There is good linearity between the luminous flux and the dimming duty cycle of the LED, owing to an $\mathrm{R}^{2}$ (coefficient of determination) value close to 1 .

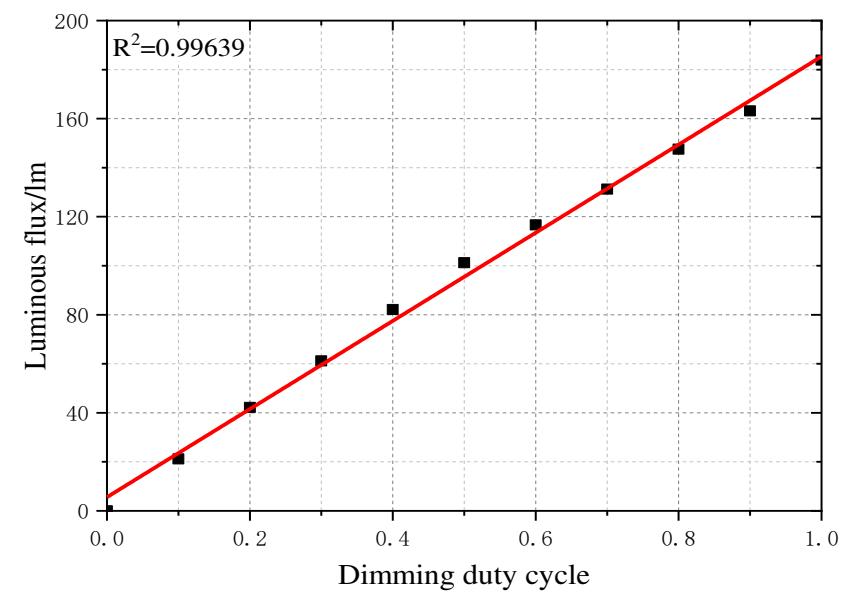

Fig. 11 Luminous flux vs. duty cycle 
The working current determines the correlated color temperature of the white light LED, which produces white light using a blue light stimulating yellow phosphor. PWM dimming is realized by a periodic on-off constant working current; therefore, the luminous flux can be adjusted while maintaining the correlated color temperature. In addition, PWM dimming is easier to realize with high precision [24]. However, PWM dimming causes LED flickering with low frequency, which would cause discomfort or even damage to human eyes. To make dimming comfortable, a high frequency, at least $1 \mathrm{kHz}$, should be adopted. The dimming frequency used in this test was $1.6 \mathrm{kHz}$.

\subsection{Test of Mixed Luminous Flux of Bi-color LED}

According to discussion in Sec. 4.1, the mixed luminous flux of a bi-color LED light source is linear and has a duty cycle $D$ of signal $\mathrm{PWM}_{1}$, which is independent of duty cycle $d$ of signal $\mathrm{PWM}_{2}$, when the luminous flux of cold light and warm light is equal. Therefore, the method used in this study can adjust the correlated color temperature as well as maintain a constant illumination. With the duty cycle $D$ of the $\mathrm{PWM}_{1}$ signal set to one, the test results and theoretical values of the mixed luminous flux are given by Table 2 . With variation of the duty cycle $d$ of the $\mathrm{PWM}_{2}$ signal, the maximum error of the mixed luminous flux between the theoretical value and the test result is $4 \%$, the minimum error is $0.22 \%$, and the average error is $1.82 \%$. Clearly, the error between the test and calculation is minimal.

Table 2 Theoretical values and test results of luminous flux

\begin{tabular}{ccccc}
\hline $\begin{array}{c}\text { duty cycle } \\
\text { /d }\end{array}$ & theory /lm & test /lm & error & error\% \\
\hline 0 & 179.69 & 179.23 & 0.46 & 0.26 \\
0.1 & 179.64 & 180.04 & 0.4 & 0.22 \\
0.2 & 179.58 & 183.62 & 4.04 & 2.25 \\
0.3 & 179.53 & 184.23 & 4.70 & 2.62 \\
0.4 & 179.48 & 186.65 & 7.17 & 4.00 \\
0.5 & 179.43 & 185.76 & 6.33 & 3.53
\end{tabular}




\begin{tabular}{ccccc}
0.6 & 179.37 & 184.63 & 5.31 & 2.96 \\
0.7 & 179.32 & 182.92 & 3.60 & 2.01 \\
0.8 & 179.26 & 180.51 & 1.25 & 0.70 \\
0.9 & 179.21 & 181.13 & 1.92 & 1.07 \\
1 & 179.16 & 179.87 & 0.71 & 0.40 \\
\hline
\end{tabular}

The test results of the mixed luminous flux with the duty cycle $D$ of $\mathrm{PWM}_{1}$ set to $0.2,0.4$, 0.6, and 0.8 are shown in Fig. 12. Given a duty cycle D, the difference of the luminous flux is small as the duty cycle $d$ varies. From the test, it can be concluded that duty cycle $d$ has no effect on the mixed luminous flux when the luminous flux of the cold light and warm light is equal.

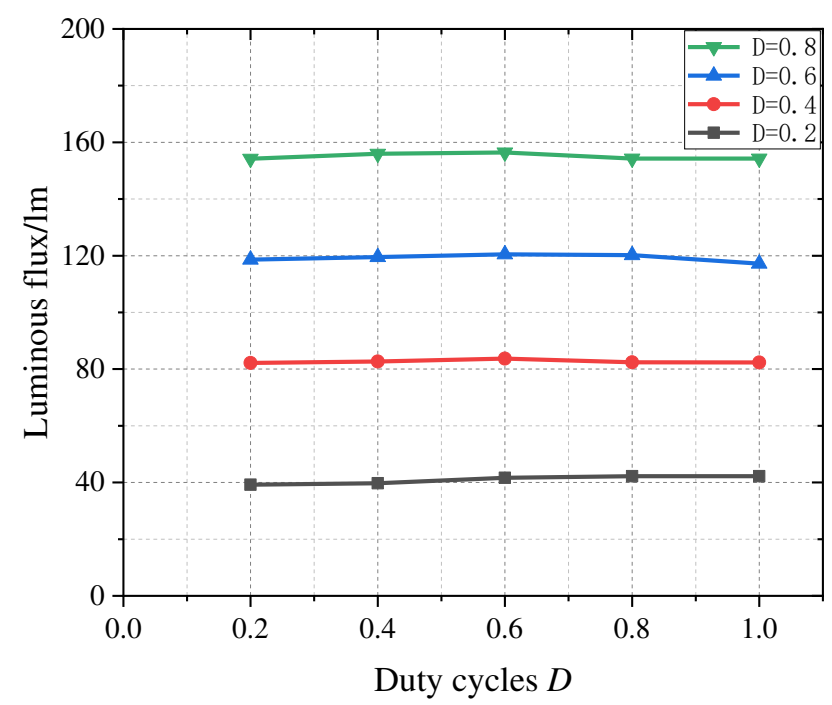

Fig. 12 Mixed luminous flux vs. duty cycles $D$ and $d$

\subsection{Test of Correlated Color Temperature}

Three group test results, the theoretical values of the correlated color temperature, and the corresponding error are given in Table 3. With the variation of the duty cycle of the cold light dimming signal, which is equal to $1-d$ according to the Fig. 3, the maximum difference between the theoretical values and the test results of the correlated color temperature is $72 \mathrm{~K}$, the minimum difference is $1 \mathrm{~K}$, and the average error is $28 \mathrm{~K}$. The results show that the method used in this study exhibits high accuracy. 
Table 3 Theoretical values and test results of correlated color temperature

\begin{tabular}{cccccccc}
\hline $\mathrm{D}_{\mathrm{C}}$ & theory /K & test1 /K & error1 /K & test2 /K & error2 /K & test3 /K & error3 /K \\
\hline 0 & 2840 & 2838 & 2 & 2841 & 1 & 2842 & 2 \\
0.1 & 3064 & 3088 & 24 & 3086 & 22 & 3090 & 26 \\
0.2 & 3331 & 3375 & 44 & 3366 & 35 & 3362 & 31 \\
0.3 & 3648 & 3671 & 23 & 3672 & 24 & 3684 & 36 \\
0.4 & 4028 & 4039 & 11 & 4039 & 11 & 4027 & 1 \\
0.5 & 4484 & 4434 & 50 & 4437 & 47 & 4455 & 29 \\
0.6 & 5035 & 5051 & 16 & 5050 & 15 & 5040 & 5 \\
0.7 & 5704 & 5722 & 18 & 5726 & 22 & 5744 & 40 \\
0.8 & 6525 & 6528 & 3 & 6482 & 43 & 6574 & 49 \\
0.9 & 7538 & 7532 & 4 & 7500 & 38 & 7492 & 46 \\
1 & 8800 & 8854 & 54 & 8864 & 64 & 8872 & 72 \\
\hline
\end{tabular}

Figure13 shows the lighting effects obtained with the variation of correlated color temperatures and a constant illuminance of $570 \mathrm{~lx}$. The power of a single light source is $3 \mathrm{~W}$, and the distance between the light source and the target is $0.6 \mathrm{~m}$. With the increase in $\mathrm{D}_{\mathrm{c}}$, the correlated color temperature changed from low to high, and the picture gradually appeared cooler.

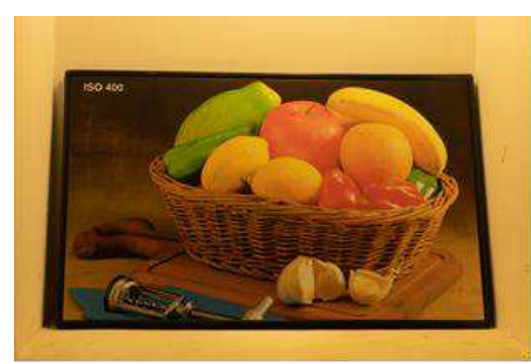

(a) $\mathrm{Dc}=0, \mathrm{CCT}=2838 \mathrm{~K}$

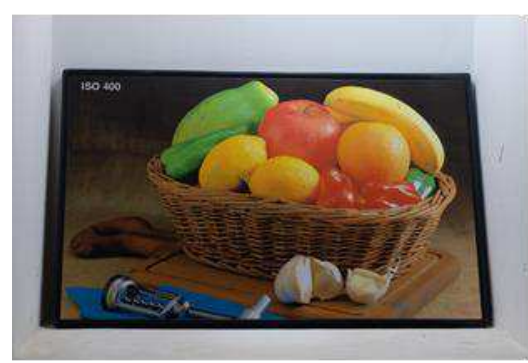

(d) $\mathrm{Dc}=0.6, \mathrm{CCT}=5051 \mathrm{~K}$

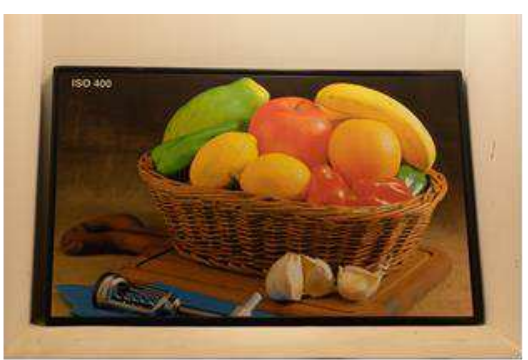

(b) $\mathrm{Dc}=0.2, \mathrm{CCT}=3375 \mathrm{~K}$

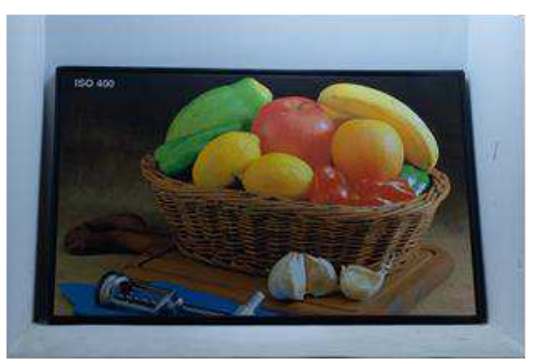

(e) $\mathrm{Dc}=0.8, \mathrm{CCT}=6528 \mathrm{~K}$

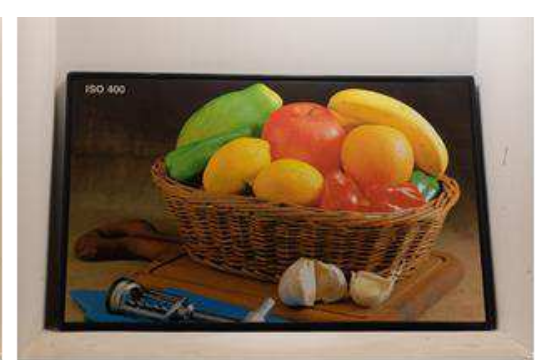

(c) $\mathrm{Dc}=0.4, \mathrm{CCT}=4039 \mathrm{~K}$

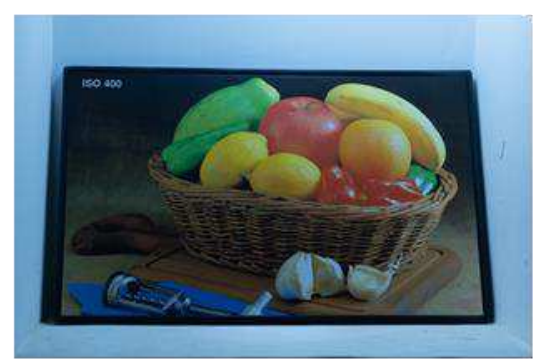

(f) $\mathrm{Dc}=1, \mathrm{CCT}=8854 \mathrm{~K}$

Fig. 13 Lighting effect with constant illumination and different correlated color temperatures 
Figure 14 shows the lighting effect with two correlated color temperatures and three illuminances; the duty cycle of $\mathrm{PWM}_{1}$ is $D$. The power of a single LED and the distance were the same as in the former experiment. The correlated color temperature of Fig. 14(a)-(c) is $3375 \mathrm{~K}$, and that of Fig. 14(d)-(f) is $6528 \mathrm{~K}$. Compared with the high correlated color temperature and high illumination Fig. 14(f), the high correlated color temperature and low illumination Fig. 14(d) looks darker and cooler.

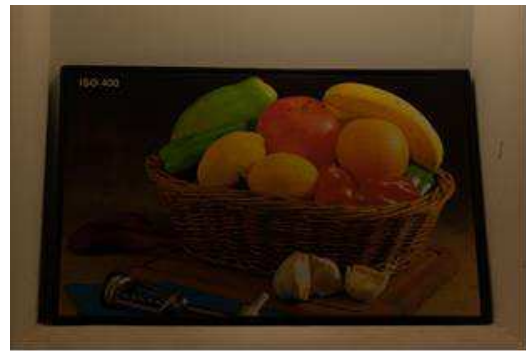

(a) $\mathrm{D}=0.2, \mathrm{E}=120 \mathrm{~lx}$

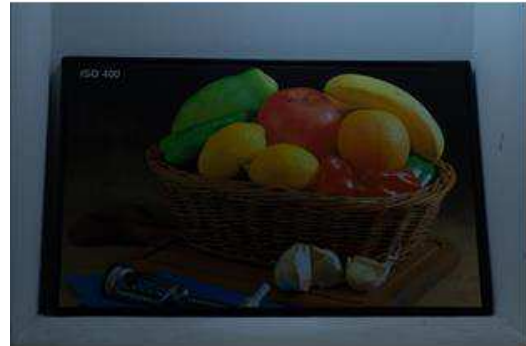

(d) $\mathrm{D}=0.2, \mathrm{E}=122 \mathrm{~lx}$

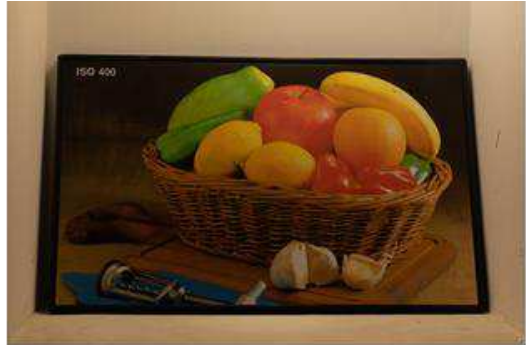

(b) $\mathrm{D}=0.5, \mathrm{E}=292 \mathrm{~lx}$

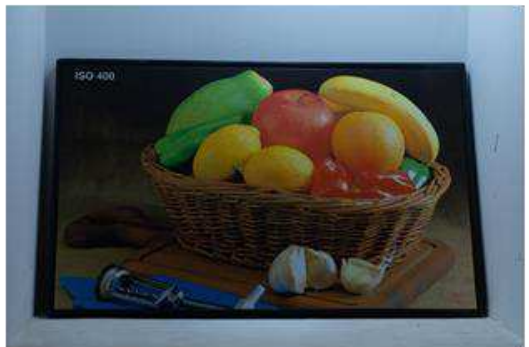

(e) $\mathrm{D}=0.5, \mathrm{E}=294 \mathrm{~lx}$

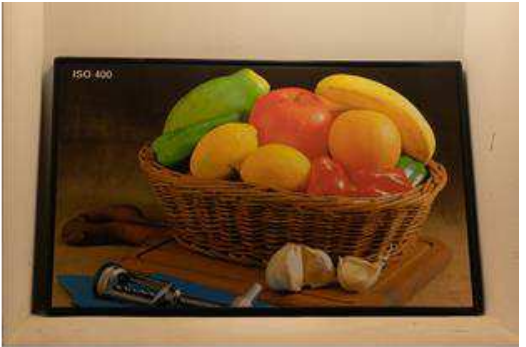

(c) $\mathrm{D}=0.8, \mathrm{E}=460 \mathrm{~lx}$

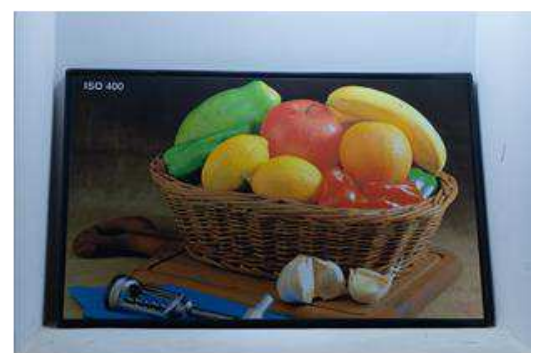

(f) $\mathrm{D}=0.8, \mathrm{E}=488 \mathrm{~lx}$

Fig. 14 Lighting effect with constant correlated color temperature and different illuminances

A low correlated color temperature matches low illumination, and high correlated color temperature matches high illumination; moreover, a reasonable combination of the correlated color temperature and illumination makes people comfortable. Figure 14(d), which has low illumination and a high color temperature, looks significantly uncomfortable compared with Fig. 14(f), which has high illumination and a high correlated color temperature. The method that we used can easily adjust the illuminance and correlated color temperature, and their combination can be obtained in accordance with the Kruithof curve. 


\section{Conclusion}

To simply color temperature adjustment of bi-color LEDs, the duty cycle of two PWM dimming signals were correlated, and the mapping relationship between the correlated color temperature and a single duty cycle was given. A bi-color LED light source system was designed with a dimming signal and color matching signal that could adjust the correlated color temperature while maintaining a constant value for the luminous flux, which makes the method convenient for application in daylight harvesting, in addition to mood lighting. Experiments showed that the method used to adjust the correlated color temperature was simple to realize within a small error. Although color temperature could be accurately adjusted within a wide range, the color rendering index of the bi-color LED light source was as poor as warm or cold LED. Improvement of the color rendering index is an important subject to investigate.

\section{Acknowledgements}

The author would like to thank the Research Institute of Photonics, Dalian Polytechnic University, China for the experimental support; Thanks to Liaoning Provincial Department of Education for funding support of scientific research project(J2020019), the anonymous reviewers and the editor for their invaluable comments and suggestions; and Editage (www.editage.cn) for English language editing.

\section{References}

[1] Zhu, Yingying1et al., "Effects of Illuminance and Correlated Color Temperature on Daytime Cognitive Performance, Subjective Mood, and Alertness in Healthy Adults," J. Environment \& Behavior.2019, Vol.51(No.2)199-230.

[2] Andersen, Marilyne1,2; Gochenour, Sharon J2; Lockley, Steven W3. "Modelling 'non-visual' effects of daylighting in a residential environment," J. Building and Environment. 2013:138-149.

[3] Münch Mirjam, Linhart Friedrich, Borisuit Apiparn, et al. "Effects of prior light exposure on early evening performance, subjective sleepiness, and hormonal secretion," 2012, 126(1):196-203.

[4] Breanne K. Hawes, Tad T. Brunyé, Caroline R. Mahoney, et al. "Effects of four workplace lighting technologies on perception, cognition and affective state," 2011, 42(1):122-128.

[5] Curcio G, Burattini C, Piccardi L, et al. "The effects of LED lighting on sleep and sleepiness," C. Congress of the European Sleep Research Society. 2016. 
[6] Seo Yoon Kang, Nara Youn, Heakyung Cecilia Yoon. "The self-regulatory power of environmental lighting: The effect of illuminance and correlated color temperature", 2019, 62:30-41.

[7] R Malik, KK Ray, S Mazumdar. "Wide-Range, Open-Loop, CCT and Illuminance Control of an LED Lamp Using Two-Component Color Blending", J. IEEE TRANSACTIONS ON POWER ELECTRONICS. 2018, 33(11): 9803-9818.

[8] Gaspare Boscarino, Mehrdad Moallem. "Daylighting Control and Simulation for LED-Based Energy-Efficient Lighting Systems," J. IEEE Transactions on Industrial Informatics. 2016, 12(1): 301-309.

[9] S. Muthu, F. J. Schuurmans, and M. D. Pashley, "Red, green, and blue LEDs for white light illumination," IEEE Trans. J. Sel. Topics Quantum Electron., 2002, 8(2): 333-338.

[10] F.-C. Wang, C.-W. Tang, and B.-J. Huang, "Multivariable robust control for a red-green-blue LED lighting system," IEEE Trans. Power Electron., 2010, 25(2): 417-428.

[11] C.-W. Tang, B.-J. Huang, and S.-P. Ying, "Illumination and color control in red-green-blue light-emitting diode," IEEE Trans. Power Electron., 2014, 29(9): 4921-4937.

[12] H. Zhao and S. W. R. Lee, "Determination of driving current of RGB LEDs for white light illumination," in Proc. 13th Int. Conf. Electron. Packag. Technol. High Density Packag., 2012. [doi:10.1109/ICEPT-HDP.2012.6474900].

[13] J. M. Gilman, M. E. Miller, and M. R. Grimaila, "A simplified control system for a daylight-matched LED lamp", J. Lighting Res. Technol., 2013, 45(5): 614-629.

[14] Lee, Albert T. L.; Chen, Huanting; Tan, Siew-Chong; Hui, S. Y. (Ron). "Precise Dimming and Color Control of LED Systems Based on Color Mixing," J. IEEE Transactions on Power Electronics.2016, Vol.31(No.1).

[15] Chew, I.1; Kalavally,V.1;Chee Pin Tan2;Parkkinen, J.3. "A Spectrally Tunable Smart LED Lighting System With Closed-Loop Control,” J. IEEE Sensors Journal.2016, Vol.16(No.11)4452-4459.

[16] Chen, Huan-Ting; Tan, Siew-Chong; Hui, S. Y. (Ron). "Nonlinear Dimming and Correlated Color Temperature Control of Bicolor White LED Systems," J. IEEE Transactions on Power Electronics.2015,Vol.30(No.12): 6934-6947.

[17] Xiu, ZR (Xiu Ziren)1; Li, H (Li Hao)1. “Application of Color Temperature Tunable LEDs in Smart Lighting System,” J. ICEPT,2014: 1423-1426.

[18] Kim H , Liu J , Jin H S , et al. "An LED color control system with independently changeable illuminance," C. International Telecommunications Energy Conference. IEEE, 2009.

[19] TAMARUS S, OHNO Y, MATSUBA Y, et al. "Measurement of color temperature: a simple and reliable method," J. Journal of Illuminating Engineering Institute of Japan, 1981, 65(4): 158-162.

[20] Xu Daisheng et al., "Dimmable light source based on cold and warm white LED," J. Journal of the optical, 2014,34 (01): 226-232.

[21] Liu Chengbi, Lu Bo, He Xin. "A novel dimming and color matching scheme to drive LED luminaire," J. LAMPS \& LIGHTING. 2014,(01):5-9.

[22] Buso S. "White Light Solid State Lamp with Luminance and Color Temperature Control," C. Brazilian Power Electronics Conference: [proceedings]. Piscataway, N. J.: IEEE, 2011: 837-843.

[23] Zhang Louying, Cui Yiping, Luo Zongnan, et al. "Color Drift in the Whole Life of High Power LED," $J$. Liquid Crystal and Display, 2010,25 (002): 210-214.

[24] Svilainis L. "LED PWM dimming linearity investigation," J. Displays, 2008, 29(3):243-249. 